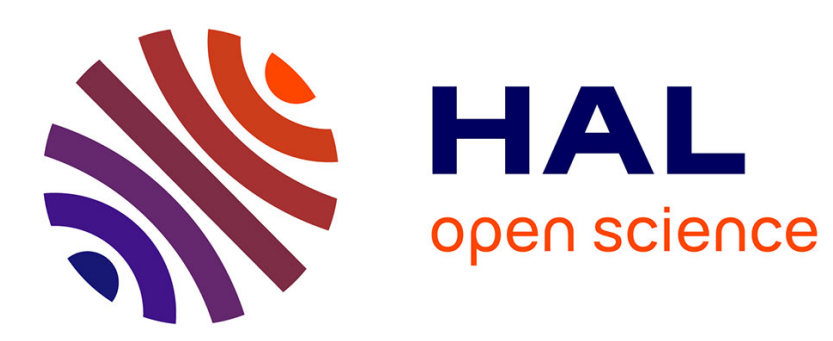

\title{
Sequential correlation based propagator algorithm for recursive subspace identification
}

Guillaume Mercère, Stéphane Lecoeuche, Christian Vasseur

\section{To cite this version:}

Guillaume Mercère, Stéphane Lecoeuche, Christian Vasseur. Sequential correlation based propagator algorithm for recursive subspace identification. 16th IFAC World Congress, IFAC'05, Jul 2005, Praha, Czech Republic. pp.CDROM. hal-00091353

\section{HAL Id: hal-00091353 https://hal.science/hal-00091353}

Submitted on 6 Sep 2006

HAL is a multi-disciplinary open access archive for the deposit and dissemination of scientific research documents, whether they are published or not. The documents may come from teaching and research institutions in France or abroad, or from public or private research centers.
L'archive ouverte pluridisciplinaire HAL, est destinée au dépôt et à la diffusion de documents scientifiques de niveau recherche, publiés ou non, émanant des établissements d'enseignement et de recherche français ou étrangers, des laboratoires publics ou privés. 


\title{
SEQUENTIAL CORRELATION BASED PROPAGATOR ALGORITHM FOR RECURSIVE SUBSPACE IDENTIFICATION
}

\author{
Guillaume Mercère ${ }^{*, * *}$ Stéphane Lecœuche ${ }^{*, * * *}$ \\ Christian Vasseur* \\ ${ }^{*}$ Laboratoire LAGIS - Bâtiment P2, USTL - 59655 Villeneuve \\ d'Ascq Cedex - France \\ ** EIPC, Campus de la Malassise - BP39 - 62967 Longuenesse \\ Cedex-France-gmercere@eipc.fr \\ ${ }^{* * *}$ Ecole des Mines de Douai - Rue Charles Bourseul - BP 838 - \\ 59508 Douai Cedex -France
}

\begin{abstract}
The problem of the recursive identification of MIMO state space models in the framework of subspace methods is considered in this article. Two new algorithms, based on a recursive formulation of the MOESP identification class, are more precisely developed. The specific feature of these methods is that they share a single algorithm to recursively estimate a basis of the observability matrix. Two propagator based (Munier and Delisle, 1991) criteria are introduced. A sequential RLS algorithm is proposed to equally minimise these cost functions. The benefits of these new techniques in comparison with EIVPAST and UDPAST (Lovera et al., 2000) are emphasized with a simulation example. Copyright ${ }^{\oplus} 2005$ IFAC.
\end{abstract}

Keywords: Identification algorithms, recursive algorithms, subspace methods, state-space models, recursive least squares.

\section{INTRODUCTION}

The development of efficient recursive subspace identification methods has been an active area of research since the end of the 90's (Gustafsson, 1998; Lovera et al., 2000; Mercère et al., 2004b). The offline subspace identification algorithms (Viberg, 1995), which are known to be an excellent alternative to the classical prediction error methods (Ljung, 1999), are indeed directly online unusable due to the application of computationally burdensome steps such as the singular value decomposition (SVD). It was also necessary to find SVD alternative algorithms in order to apply the subspace concept in a recursive framework. Two points of view were proposed to solve this problem:

- The first one consists in adapting the array signal processing Yang's criterion (Yang, 1995) to the recursive update of the observability matrix. Several algorithms have been developed (Gustafsson, 1998; Lovera et al., 2000; Lovera, 2003). All of them work in the presence of measurement and process noises thanks to the introduction of instrumental variables.

- The second one rests on the adaptation of an other array signal processing technique: the propagator method (Munier and Delisle, 1991). The advantage of this approach over the previous conception lies in the use of a linear operator and unconstrained and unapproximated quadratic criteria which lead to easy recursive least squares techniques (Mercère et al., 2003).

The algorithms proposed in this paper are based on this last concept. As any recursive subspace identification method (cf. §2), they are made up of two stages: 
- the online update of a particular vector named the observation vector;

- the recursive estimation of the observability matrix from the online update of the propagator.

However, contrary to the SVD alternative techniques developed so far, they use a single recursive propagator estimation algorithm equally applicable whatever the noise properties of the observation vector are. The basis of this algorithm is found in the works of J. L. Yu on a particular correlation-based projection approximation subspace tracking technique (Yu, 2000).

The outline of this paper is as follows: after a short description of the analogy between recursive subspace identification and subspace tracking in array signal processing (cf. §2), the problem of the recursive observation vector estimation is considered in section 3 thanks to the use of specific QR factorization updates. The recursive estimation of a basis of the observability subspace is then proposed in section 4. Two different quadratic criteria and a single RLS sequential algorithm are more particularly developed. The performances of this new algorithm are finally shown on a simulation example.

\section{ANALOGY BETWEEN RECURSIVE SUBSPACE IDENTIFICATION AND SUBSPACE TRACKING IN ARRAY SIGNAL PROCESSING}

In recursive subspace identification, the goal is to estimate online the $[\mathbf{A}, \mathbf{B}, \mathbf{C}, \mathbf{D}]$ system matrices of the following noisy state space representation:

$$
\begin{aligned}
\mathbf{x}(t+1) & =\mathbf{A} \mathbf{x}(t)+\mathbf{B} \tilde{\mathbf{u}}(t)+\mathbf{w}(t) \\
\tilde{\mathbf{y}}(t) & =\mathbf{C x}(t)+\mathbf{D} \tilde{\mathbf{u}}(t) \\
\mathbf{y}(t) & =\tilde{\mathbf{y}}(t)+\mathbf{v}(t) \\
\mathbf{u}(t) & =\tilde{\mathbf{u}}(t)+\mathbf{f}(t) .
\end{aligned}
$$

where the noise-free inputs $\tilde{\mathbf{u}} \in \mathbb{R}^{n_{u} \times 1}$ and outputs $\tilde{\mathbf{y}} \in \mathbb{R}^{n_{y} \times 1}$ are disturbed by process and measurement white noises, respectively named $\mathbf{w}, \mathbf{f}$ and $\mathbf{v}$.

In subspace tracking in array signal processing (Krim and Viberg, 1996), the considered problem consists in recursively determining the directions of arrival $\boldsymbol{\theta}$ by estimating the steering matrix $\boldsymbol{\Gamma}(\boldsymbol{\theta})$ from the following data generation model:

$$
\mathbf{z}(t)=\Gamma(\boldsymbol{\theta}) \mathbf{s}(\mathbf{t})+\mathbf{b}(t)
$$

where $\mathbf{z}$ is the output of the $n_{z}$ sensors of the antenna array, $\mathbf{s}$ the vector of the $n_{s}$ signal waveforms and $\mathbf{b}$ the additive noise.

When studying the main techniques developed in both fields, it is interesting to note that the mathematical problem is the same: to track some eigencomponents of particular matrices by adapting specific subspaces with the last observations. Now, in array signal processing, notable algorithms have been developed to avoid the use of eigenvalue decomposition (Krim and Viberg, 1996). Thus, it seems to be interesting to adapt some of these SVD alternatives for recursive subspace identification. For that, it is initially essential to rewrite the state space system (1) in an equivalent way to the model (2). This last equation contains an output vector $\mathbf{z}$ composed by spatially stacked signals. Now, in identification, the available data are only temporal observations. It is also necessary to introduce a temporal window similar to the spatial one used in subspace tracking:

$$
\mathbf{y}_{f}^{+}(t)=\left[\begin{array}{llll}
\mathbf{y}^{T}(t) & \cdots & \mathbf{y}^{T}(t+f-1)
\end{array}\right]^{T} \in \mathbb{R}^{n_{y} f \times 1}
$$

where $f>n_{x}$ is a user fixed integer. It is easy to verify the following relation ${ }^{1}$ :

$$
\begin{aligned}
\mathbf{y}_{f}^{+}(t)=\boldsymbol{\Gamma}_{f} \mathbf{x}(t) & +\underbrace{\mathbf{H}_{f} \mathbf{u}_{f}^{+}(t)}_{\mathbf{b}_{f}^{+}(t)} \\
& +\underbrace{\mathbf{G}_{j} .}_{\mathbf{m}_{f} \mathbf{w}_{f}^{+}(t)-\mathbf{H}_{f} \mathbf{f}_{f}^{+}(t)+\mathbf{v}_{f}^{+}(t)}
\end{aligned}
$$

where $\Gamma_{f}$ is the observability matrix:

$$
\boldsymbol{\Gamma}_{f}=\left[\begin{array}{llll}
\mathbf{C}^{T}(\mathbf{C A})^{T} & \cdots & \left(\mathbf{C A}^{f-1}\right)^{T}
\end{array}\right]^{T} .
$$

The connection between subspace identification and array signal processing becomes apparent by writing:

$$
\mathbf{z}_{f}^{+}(t)=\mathbf{y}_{f}^{+}(t)-\mathbf{H}_{f} \mathbf{u}_{f}^{+}(t)=\Gamma_{f} \mathbf{x}(t)+\mathbf{b}_{f}^{+}(t) .
$$

This relation stresses on both steps required to recursively estimate the system matrices ${ }^{2}[\mathbf{A}, \mathbf{B}, \mathbf{C}, \mathbf{D}]$ :

(1) the update of the "observation vector" $\mathbf{z}_{f}^{+}$from the noisy input output measurements:

$$
\mathbf{z}_{f}^{+}(t)=\mathbf{y}_{f}^{+}(t)-\mathbf{H}_{f} \mathbf{u}_{f}^{+}(t),
$$

(2) the estimation of a basis of $\boldsymbol{\Gamma}_{f}$ from this observation vector:

$$
\mathbf{z}_{f}^{+}(t)=\boldsymbol{\Gamma}_{f} \mathbf{x}(t)+\mathbf{b}_{f}^{+}(t) .
$$

Both stages will be considered in the following sections. The observability matrix determination phase is more precisely studied in this article. It is however necessary to obtain an accurate estimate of the observation vector in a first step. This problem is analysed in the next section.

\section{RECURSIVE OBSERVATION VECTOR ESTIMATION}

The first stage of any recursive subspace identification techniques lies on the observation vector estimation at each new acquisition. The proposed approach, initially developed by M. Lovera (Lovera et al., 2000), consists in updating the QR factorization of two MOESP schemes: the Ordinary MOESP (Verhaegen and Dewilde, 1992) and the PI/PO MOESP (Verhaegen, 1994). This mathematical tool indeed allows to remove (Viberg, 1995) the column subspace spanned

\footnotetext{
1 The stacked vectors of the input and noises are defined in the same way than $\mathbf{y}_{f}^{+} \cdot \mathbf{G}_{f}$ and $\mathbf{H}_{f}$ are two Toeplitz matrices.

2 Notice that $\mathbf{H}_{f}$ matrix is unknown at time $t$ since the state space matrices used in its construction are still not estimated.
} 
by $\mathbf{H}_{f}$ from the column subspace spanned by the $\mathbf{Y}_{f}^{+}$ Hankel matrix defined as:

$$
\mathbf{Y}_{f}^{+}(\bar{t})=\left[\begin{array}{llll}
\mathbf{y}_{f}^{+}(t) & \cdots & \mathbf{y}_{f}^{+}(t+N-1)
\end{array}\right]
$$

with $N>>f>n_{x}$ and $\bar{t}=t+N-1$. It is also interesting to update these decompositions at each new input-output measurement to recursively calculate the subtraction $\mathbf{y}_{f}^{+}-\mathbf{H}_{f} \mathbf{u}_{f}^{+}$. These update schemes are presented in the next two subsections.

\subsection{The recursive Ordinary MOESP update}

The basic idea of this approach is to consider the QR decomposition of the offline Ordinary MOESP scheme and its update at each new acquisition. For that purpose, consider the following QR factorization:

$$
\left[\begin{array}{c}
\mathbf{U}_{f}^{+}(\bar{t}) \\
\mathbf{Y}_{f}^{+}(\bar{t})
\end{array}\right]=\left[\begin{array}{cc}
\mathbf{R}_{11}(\bar{t}) & \mathbf{0} \\
\mathbf{R}_{21}(\bar{t}) & \mathbf{R}_{22}(\bar{t})
\end{array}\right]\left[\begin{array}{l}
\mathbf{Q}_{1}(\bar{t}) \\
\mathbf{Q}_{2}(\bar{t})
\end{array}\right] .
$$

When a new input-output couple $\{\mathbf{u}(\bar{t}+1), \mathbf{y}(\bar{t}+1)\}$ is acquired, this decomposition can be updated as ${ }^{3}$ :

$$
\left[\sqrt{\lambda}\left[\begin{array}{cc}
\mathbf{R}_{11}(\bar{t}) & \mathbf{0} \\
\mathbf{R}_{21}(\bar{t}) & \mathbf{R}_{22}(\bar{t})
\end{array}\right] \begin{array}{c}
\mathbf{u}_{f}^{+}(\bar{t}+1) \\
\mathbf{y}_{f}^{+}(\bar{t}+1)
\end{array}\right]\left[\begin{array}{cc}
\mathbf{Q}_{1}(\bar{t}) & \mathbf{0} \\
\mathbf{Q}_{2}(\bar{t}) & \mathbf{0} \\
\mathbf{0} & 1
\end{array}\right] .
$$

A sequence of Givens rotations (Golub and Van Loan, 1996) can then be used to annihilate the stacked input vector $\mathbf{u}_{f}^{+}$and bring back the $\mathbf{R}$ factor to the following block lower triangular form:

$$
\left[\begin{array}{ccc}
\mathbf{R}_{11}(\bar{t}+1) & \mathbf{0} & \mathbf{0} \\
\mathbf{R}_{21}(\bar{t}+1) & \sqrt{\lambda} \mathbf{R}_{22}(\bar{t}) & \overline{\mathbf{z}}_{f}^{+}(\bar{t}+1)
\end{array}\right] .
$$

$\overline{\mathbf{z}}_{f}^{+}$is the vector obtained by modifying $\mathbf{y}_{f}^{+}$in order to include the information contained in $\mathbf{u}_{f}^{+}$and $\left[\begin{array}{ll}\mathbf{R}_{11}^{T} & \mathbf{R}_{21}^{T}\end{array}\right]^{T}$. Then, it is possible to prove that (Mercère et al., 2004b):

$$
\overline{\mathbf{z}}_{f}^{+}(\bar{t}+1)= \pm \mathbf{z}_{f}^{+}(\bar{t}+1) .
$$

Remark 1. In practice, there is no need to update $\mathbf{R}_{22}$. $\mathbf{R}_{11}$ and $\mathbf{R}_{21}$ are only used to estimate $\mathbf{z}_{f}^{+}$. So, the theoretical growing size of the $\mathbf{R}$ factor is not a problem in practice. The computational complexity of this partial QR factorization update is $\mathscr{O}\left(\left(\frac{n_{u}}{2}+n_{y}\right) n_{u} f^{2}\right)$.

This technique leads to unbiased estimates only if the output measurement noise $\mathbf{v}$ is white and the other disturbances are null. Since this condition is too restrictive, the development of a recursive PI/PO MOESP update algorithm has been proposed to estimate an asymptotically noise purged observation vector.

\subsection{The recursive $P I / P O$ MOESP update}

The principle of the proposed method is based on the same idea as in the previous paragraph i.e. updating

\footnotetext{
3 The forgetting factor $\lambda$ is introduced to weight the past informations.
}

the P I / PO MOESP QR factorization at each time step by applying Givens rotations. This second technique has the advantage to treat the problem of the noise by using suitable instrumental variables. Thus, consider the following QR factorization:

$$
\left[\begin{array}{c}
\mathbf{U}_{f}^{+}(\bar{t}) \\
\mathbf{\Xi}(\bar{t}) \\
\mathbf{Y}_{f}^{+}(\bar{t})
\end{array}\right]=\left[\begin{array}{ccc}
\mathbf{R}_{11}(\bar{t}) & \mathbf{0} & \mathbf{0} \\
\mathbf{R}_{21}(\bar{t}) & \mathbf{R}_{22}(\bar{t}) & \mathbf{0} \\
\mathbf{R}_{31}(\bar{t}) & \mathbf{R}_{32}(\bar{t}) & \mathbf{R}_{33}(\bar{t})
\end{array}\right]\left[\begin{array}{l}
\mathbf{Q}_{1}(\bar{t}) \\
\mathbf{Q}_{2}(\bar{t}) \\
\mathbf{Q}_{3}(\bar{t})
\end{array}\right]
$$

where $\mathbf{\Xi}=\left[\mathbf{U}_{p}^{-T} \mathbf{Y}_{p}^{-T}\right]^{T}$ in the PO scheme and $\boldsymbol{\Xi}=$ $\mathbf{U}_{p}^{-}$in the PI scheme with ${ }^{4}$ :

$$
\begin{aligned}
\mathbf{U}_{p}^{-}(\bar{t}) & =\left[\begin{array}{lll}
\mathbf{u}_{p}^{-}(t) & \cdots & \mathbf{u}_{p}^{-}(t+N-1)
\end{array}\right] \\
\mathbf{Y}_{p}^{-}(\bar{t}) & =\left[\begin{array}{lll}
\mathbf{y}_{p}^{-}(t) & \cdots & \mathbf{y}_{p}^{-}(t+N-1)
\end{array}\right] \\
\mathbf{u}_{p}^{-}(t) & =\left[\begin{array}{lll}
\mathbf{u}^{T}(t-p) & \cdots & \mathbf{u}^{T}(t-1)
\end{array}\right]^{T} \\
\mathbf{y}_{p}^{-}(t) & =\left[\begin{array}{lll}
\mathbf{y}^{T}(t-p) & \cdots & \mathbf{y}^{T}(t-1)
\end{array}\right]^{T} .
\end{aligned}
$$

When new input-output data are available, this decomposition can be updated as:

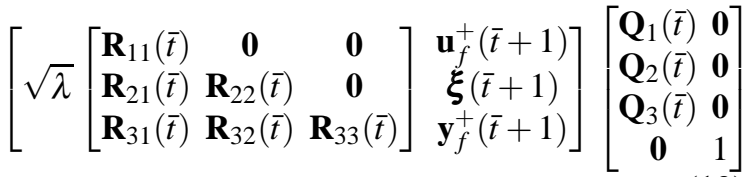

with $\boldsymbol{\xi}(\bar{t}+1)=\mathbf{u}_{p}^{-}(\bar{t}+1)$ for the PI MOESP version and $\boldsymbol{\xi}(\bar{t}+1)=\left[\mathbf{u}_{p}^{-T}(\bar{t}+1) \mathbf{y}_{p}^{-T}(\bar{t}+1)\right]^{T}$ for $\mathrm{PO}$ MOESP. Givens rotations are then used twice to update this factorization. They are first applied to put the elements of $\mathbf{u}_{f}^{+}(\bar{t}+1)$ at zero:

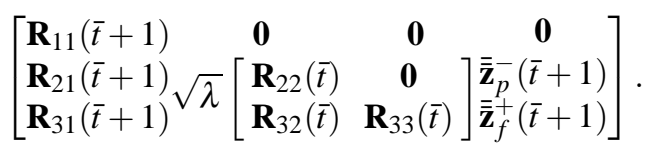

A second sequence of Givens rotations is then employed to annihilate $\overline{\mathbf{z}}_{p}^{-}(\bar{t}+1)$ and leads to:

$$
\left[\begin{array}{cccc}
\mathbf{R}_{11}(\bar{t}+1) & \mathbf{0} & \mathbf{0} & \mathbf{0} \\
\mathbf{R}_{21}(\bar{t}+1) & \mathbf{R}_{22}(\bar{t}+1) & \mathbf{0} & \mathbf{0} \\
\mathbf{R}_{31}(\bar{t}+1) & \mathbf{R}_{32}(\bar{t}+1) & \sqrt{\lambda} \mathbf{R}_{33}(\bar{t}) & \overline{\overline{\mathbf{z}}}_{f}^{+}(\bar{t}+1)
\end{array}\right] .
$$

By following the proof given $\$ 4.1$ in (Mercère et al., 2004b), it is possible to show that:

$$
\begin{aligned}
& \quad \mathbf{z}_{f}^{+}(\bar{t}+1) \mathbf{z}_{f}^{+T}(\bar{t}+1)= \\
& \overline{\overline{\mathbf{z}}}_{f}^{+}(\bar{t}+1) \overline{\overline{\mathbf{z}}}_{f}^{+T}(\bar{t}+1)-\overline{\overline{\mathbf{z}}}_{f}^{+}(\bar{t}+1) \overline{\overline{\mathbf{z}}}_{f}^{+T}(\bar{t}+1) .
\end{aligned}
$$

The estimated matrix $\overline{\overline{\mathbf{z}}}_{f}^{+} \overline{\overline{\mathbf{z}}}_{f}^{+T}-\overline{\overline{\mathbf{z}}}_{f}^{+} \overline{\overline{\mathbf{z}}}_{f}^{+}$is in fact asymptotically rid of the noise effects. Then, it is possible to update, at each step, the noise free observation covariance matrix:

$$
\begin{aligned}
& \mathbf{R}_{\tilde{\mathbf{z}}_{f}^{+}}(\bar{t}+1)=\lambda \mathbf{R}_{\tilde{\mathbf{z}}_{f}^{+}}(\bar{t})+ \\
& \overline{\overline{\mathbf{z}}}_{f}^{+}(\bar{t}+1) \overline{\overline{\mathbf{z}}}_{f}^{+T}(\bar{t}+1)-\overline{\overline{\mathbf{z}}}_{f}^{+}(\bar{t}+1) \overline{\overline{\mathbf{z}}}_{f}^{+T}(\bar{t}+1) .
\end{aligned}
$$


Remark 2. As previously, there is no need to totally complete the PI/PO MOESP QR factorization in practice. The complexity of this update technique is however larger: $\mathscr{O}\left(\left(\frac{n_{u} f+n_{\xi}}{2}+n_{y} f\right)\left(n_{u} f+n_{\xi}\right)\right)$.

\section{RECURSIVE UPDATE OF THE OBSERVABILITY MATRIX}

In the previous section, it has been shown it is possible to update the observation vector by two different ways according to the hypotheses on the disturbances acting on the system. From now on, consider the most important step in recursive subspace identification: the recursive estimation of the observability matrix. Like in (Gustafsson, 1998; Lovera et al., 2000; Lovera, 2003), it is proposed to exploit the close relationship between array signal processing and subspace identification to suggest efficient SVD alternatives. Since the recursive identification algorithms based on the propagator (Munier and Delisle, 1991) have shown several advantages compared with the algorithms adapted from PAST (Mercère et al., 2003; Mercère et al., 2004a), the approach analysed in this article rests on the propagator concept. The aim of this section consists more precisely in developing a single recursive algorithm able to minimise two different quadratic criteria. In order to introduce this algorithm, it is first of all necessary to give a brief overview of the propagator principle in the identification framework.

\subsection{Overview of the propagator method}

Assume that the studied system is observable. Since $\boldsymbol{\Gamma}_{f} \in \mathbf{R}^{n_{y} f \times n_{x}}$ with $n_{y} f>n_{x}, \boldsymbol{\Gamma}_{f}$ has at least $n_{x}$ rows, gathered in a submatrix $\Gamma_{f_{1}}$, linearly independent. Then, the complement $\Gamma_{f_{2}}$ of $\Gamma_{f_{1}}$ can be expressed as a linear combination of these $n_{x}$ rows. So, there is a unique linear operator $\mathbf{P}_{f} \in \mathbf{R}^{n_{x} \times\left(n_{y} f-n_{x}\right)}$ named propagator (Munier and Delisle, 1991) defined as:

$$
\Gamma_{f_{2}}=\mathbf{P}_{f}^{T} \boldsymbol{\Gamma}_{f_{1}} .
$$

Furthermore, it is easy to verify that:

$$
\boldsymbol{\Gamma}_{f}=\left[\begin{array}{l}
\boldsymbol{\Gamma}_{f_{1}} \\
\boldsymbol{\Gamma}_{f_{2}}
\end{array}\right]=\left[\begin{array}{c}
\boldsymbol{\Gamma}_{f_{1}} \\
\mathbf{P}_{f}^{T} \boldsymbol{\Gamma}_{f_{1}}
\end{array}\right]=\left[\begin{array}{c}
\mathbf{I}_{n_{x}} \\
\mathbf{P}_{f}^{T}
\end{array}\right] \boldsymbol{\Gamma}_{f_{1}}=\mathbf{E}_{o} \boldsymbol{\Gamma}_{f_{1}} .
$$

Thus, since $\operatorname{rank}\left\{\boldsymbol{\Gamma}_{f_{1}}\right\}=n_{x}$,

$$
\operatorname{Im}_{\text {col }}\left\{\boldsymbol{\Gamma}_{f}\right\}=\operatorname{Im}_{\text {col }}\left\{\mathbf{E}_{o}\right\} .
$$

This last equation implies that it is possible to get an expression of the observability matrix in a particular basis by determining the propagator. This operator can be estimated from the equation (8). Indeed, after having applied a data reorganization so that the first $n_{x}$ rows of $\Gamma_{f}$ are linearly independent, the following partition can be introduced:

$$
\mathbf{z}_{f}^{+}(t)=\left[\begin{array}{c}
\mathbf{z}_{f_{1}}^{+}(t) \\
\mathbf{z}_{f_{2}}^{+}(t)
\end{array}\right]=\left[\begin{array}{l}
\mathbf{I}_{n_{x}} \\
\mathbf{P}_{f}^{T}
\end{array}\right] \boldsymbol{\Gamma}_{f_{1}} \mathbf{x}(t)+\left[\begin{array}{l}
\mathbf{b}_{f_{1}}^{+}(t) \\
\mathbf{b}_{f_{2}}^{+}(t)
\end{array}\right]
$$

where $\mathbf{z}_{f_{1}}^{+}$and $\mathbf{z}_{f_{2}}^{+}$are the components of $\mathbf{z}_{f}^{+}$respectively corresponding to the $n_{x}$ rows of $\Gamma_{f_{1}}$ et $n_{y} f-n_{x}$ rows of $\boldsymbol{\Gamma}_{f_{2}}$. In the ideal noise free case, it is easy to show that:

$$
\mathbf{z}_{f_{2}}^{+}=\mathbf{P}_{f}^{T} \mathbf{z}_{f_{1}}^{+} .
$$

In the presence of noise, this relation holds no longer. An estimate of $\mathbf{P}_{f}$ can however be obtained by minimising the following cost function:

$$
J\left(\mathbf{P}_{f}\right)=E\left\|\mathbf{z}_{f_{2}}^{+}-\mathbf{P}_{f}^{T} \mathbf{z}_{f_{1}}^{+}\right\|^{2} .
$$

Unfortunately, even if the noise is spatially and temporally white, the estimate of $\mathbf{P}_{f}$ is biased. Indeed, since the asymptotic least square estimate of $\mathbf{P}_{f}^{T}$ is given by:

$$
\hat{\mathbf{P}}_{f}^{T}=\mathbf{R}_{\mathbf{z}_{f_{2}}^{+} \mathbf{z}_{f_{1}}^{+}} \mathbf{R}_{\mathbf{z}_{f_{1}}^{+}}^{-1}
$$

with $^{5}$ :

$$
\begin{aligned}
\mathbf{R}_{\mathbf{z}_{f_{2}}^{+} \mathbf{z}_{f_{1}}^{+}} & =\mathbf{P}_{f}^{T} \boldsymbol{\Gamma}_{f_{1}} \mathbf{R}_{\mathbf{x}} \Gamma_{f_{1}}^{T}+\mathbf{R}_{\mathbf{b}_{f_{2}}^{+} \mathbf{b}_{f_{1}}^{+}} \\
\mathbf{R}_{\mathbf{z}_{f_{1}}^{+}} & =\boldsymbol{\Gamma}_{f_{1}} \mathbf{R}_{\mathbf{x}} \boldsymbol{\Gamma}_{f_{1}}^{T}+\mathbf{R}_{\mathbf{b}_{f_{1}}^{+}},
\end{aligned}
$$

the estimate of $\mathbf{P}_{f}^{T}$ verifies in the white noise case:

$$
\hat{\mathbf{P}}_{f}^{T}=\mathbf{P}_{f}^{T} \boldsymbol{\Gamma}_{f_{1}} \mathbf{R}_{\mathbf{x}} \boldsymbol{\Gamma}_{f_{1}}^{T}\left(\boldsymbol{\Gamma}_{f_{1}} \mathbf{R}_{\mathbf{x}} \boldsymbol{\Gamma}_{f_{1}}^{T}+\sigma \mathbf{I}_{n_{x}}\right)^{-1}
$$

which is not equal to $\mathbf{P}_{f}^{T}$. Now, since in most of the practical situations the data are disturbed by noise, it is essential to propose other criteria in order to circumvent this difficulty. Two solutions can be considered according to the properties of the observation vector:

- If the observation vector is estimated from the recursive PI/PO MOESP update scheme (cf. $\S 3.2)$ and since ${ }^{6}$ :

$$
\mathbf{R}_{\tilde{\mathbf{z}}_{f}^{+}}=\left[\begin{array}{cc}
\mathbf{R}_{\tilde{\mathbf{z}}_{f_{1}}^{+}} & \mathbf{R}_{\tilde{\mathbf{z}}_{f_{1}}^{+} \tilde{\mathbf{z}}_{f_{2}}^{+}} \\
\mathbf{R}_{\tilde{\mathbf{z}}_{f_{2}}^{+} \tilde{\mathbf{z}}_{f_{1}}^{+}} & \mathbf{R}_{\tilde{\mathbf{z}}_{f_{2}}^{+}}
\end{array}\right]=\left[\begin{array}{cc}
\mathbf{R}_{\overline{\mathbf{x}}} & \mathbf{R}_{\overline{\mathbf{x}}} \mathbf{P}_{f} \\
\mathbf{P}_{f}^{T} \mathbf{R}_{\overline{\mathbf{x}}} & \mathbf{P}_{f}^{T} \mathbf{R}_{\overline{\mathbf{x}}} \mathbf{P}_{f}
\end{array}\right]
$$

with $\overline{\mathbf{x}}=\Gamma_{f_{1}} \mathbf{x}$, then:

$$
\hat{\mathbf{P}}_{f}^{T}=\mathbf{R}_{\tilde{\mathbf{z}}_{f_{2}}^{+}} \tilde{\mathbf{z}}_{f_{1}^{+}}^{+} \mathbf{R}_{\tilde{\mathbf{z}}_{f_{1}}^{+}}^{-1}=\mathbf{P}_{f}^{T} .
$$

This estimate is determined by minimising the following criterion:

$$
\breve{J}\left(\mathbf{P}_{f}\right)=\left\|\mathbf{R}_{\tilde{\mathbf{z}}_{f_{2}}^{+} \tilde{\mathbf{z}}_{f_{1}}^{+}}-\mathbf{P}_{f}^{T} \mathbf{R}_{\tilde{\mathbf{z}}_{f_{1}}^{+}}\right\|_{F}^{2} .
$$

- If the observation vector is estimated from the recursive Ordinary MOESP update scheme and since this estimate is biased if, for instance, $\mathbf{v}$ is not white, an instrumental variable must be introduced in the criterion (29) so as to make it applicable even if coloured disturbances act on the system (Mercère et al., 2003):

$$
J_{I V}\left(\mathbf{P}_{f}\right)=\left\|\mathbf{R}_{\mathbf{z}_{f_{2}}^{+}} \boldsymbol{\xi}-\mathbf{P}_{f}^{T} \mathbf{R}_{\mathbf{z}_{f_{1}}^{+}} \boldsymbol{\xi}\right\|_{F}^{2} .
$$

\footnotetext{
$5 \mathbf{R}_{\mathbf{a b}}$ is the cross correlation matrix of $\mathbf{a}$ and $\mathbf{b} . \mathbf{R}_{\mathbf{a}}$ is the auto correlation matrix of $\mathbf{a}$

6 the recursive PI/PO MOESP update scheme leads to an asymptotically noise free observation covariance matrix.
} 
$\boldsymbol{\xi} \in \mathbb{R}^{n_{\xi} \times 1}\left(n_{\xi} \geq n_{x}\right)$ is the instrumental variable assumed to be uncorrelated with the noise but sufficiently correlated with the state vector $\mathbf{x}$.

The cost functions (36) and (37) can be minimised by different ways. Several algorithms have been developed to estimate $\mathbf{P}_{f}$ from the criterion (37) according to the number of instruments in $\boldsymbol{\xi}$ (Mercère et $a l ., 2003$; Mercère et al., 2004b). It is however important to note that both criteria $J_{I V}$ and $\breve{J}$ have the same structure. It would be interesting to develop a single algorithm which is able to minimise both cost functions. This one is introduced in the following subsection.

4.2 A single algorithm to recursively estimate the propagator: the sequential correlation based propagator method

Consider the following generalised criterion:

$$
\bar{J}=\left(\mathbf{P}_{f}\right)=\left\|\mathbf{R}_{2}-\mathbf{P}_{f}^{T} \mathbf{R}_{1}\right\|_{F}^{2}
$$

where $\mathbf{R}_{2}$ and $\mathbf{R}_{1}$ are respectively $\mathbf{R}_{\mathbf{z}_{f_{2}}^{+} \boldsymbol{\xi}}$ and $\mathbf{R}_{\mathbf{z}_{f_{1}}^{+} \boldsymbol{\xi}}$ when $J_{I V}$ is taken into account and $\mathbf{R}_{\tilde{\mathbf{z}}_{f_{2}}^{+}} \tilde{\mathbf{z}}_{f_{1}}^{+}$and $\mathbf{R}_{\tilde{\mathbf{z}}_{f_{1}}^{+}}$ when $\breve{J}$ is considered. It is then possible to minimise this criterion by taking inspiration in the works of J. L. Yu (Yu, 2000) on the PAST algorithms (Yang, 1995 ) in array signal processing. This author proposes to estimate the signal subspace by minimising the following cost function ${ }^{7}$ :

$$
V(\mathbf{W})=\left\|\mathbf{R}-\mathbf{W} \mathbf{W}^{T} \mathbf{R}\right\|_{F}^{2}
$$

by sequentially minimising $n_{z}$ criteria defined as:

$$
V_{i}(\mathbf{W}(t))=\sum_{k=1}^{t} \lambda^{t-k}\left\|\mathbf{r}^{i}(k)-\mathbf{W}(t) \mathbf{W}^{T}(t) \mathbf{r}^{i}(k)\right\|^{2}
$$

where $\mathbf{r}^{i}$ is the $i$ th column of $\mathbf{R}$. According to J. L. $\mathrm{Yu}$ (Yu, 2000), this sequential procedure converges towards the global minimum of (39) (which is equal to $\mathbf{U}_{s} \mathbf{T}$ where $\mathbf{U}_{s}$ contains the $n_{s}$ dominating eigenvectors of $\mathbf{R}$ and $\mathbf{T}$ is an arbitrary unitary matrix) when the noise $\mathbf{b}$ (cf. equ. (2)) is spatially and temporally white.

In our case, since we respectively consider that:

- either a noise free estimate $\mathbf{R}_{\tilde{\mathbf{z}}_{f}^{+}}$can be obtained

- or an instrumental variable is introduced to annihilate the noise effect,

the following criteria $\bar{J}_{i}, i \in\left[1, n_{c}\right]$, can be used to estimate the propagator:

$$
\bar{J}_{i}\left(\mathbf{P}_{f}(t)\right)=\sum_{k=1}^{t} \lambda^{t-k}\left\|\mathbf{r}_{2}^{i}(k)-\mathbf{P}_{f}^{T}(t) \mathbf{r}_{1}^{i}(k)\right\|^{2} .
$$

$\mathbf{r}_{2}^{i}$ and $\mathbf{r}_{1}^{i}$ are the $i$ th column of $\mathbf{R}_{2}$ and $\mathbf{R}_{1} \cdot n_{c}$ is the number of columns of $\mathbf{R}_{1}$. Since these cost functions are directly quadratic in $\mathbf{P}_{f}$, each of them can

\footnotetext{
$7 \mathbf{W} \in \mathbb{C}^{n_{z} \times n_{s}}$ and $\mathbf{R}$ is the covariance matrix of the observations.
}

be minimised by a recursive least squares procedure (Ljung, 1999):

$$
\begin{aligned}
\mathbf{K}(t) & =\frac{\mathbf{r}_{1}^{i^{T}}(t) \mathbf{L}(t-1)}{\lambda+\mathbf{r}_{1}^{i^{T}}(t) \mathbf{L}(t-1) \mathbf{r}_{1}^{i}(t)} \\
\mathbf{L}(t) & =\frac{1}{\lambda}\left(\mathbf{L}(t-1)-\mathbf{L}(t-1) \mathbf{r}_{1}^{i}(t) \mathbf{K}(t)\right) \\
\mathbf{P}_{f}^{T}(t) & =\mathbf{P}_{f}^{T}(t-1)+\left(\mathbf{r}_{2}^{i}(t)-\mathbf{P}_{f}^{T}(t-1) \mathbf{r}_{1}^{i}(t)\right) \mathbf{K}(t) .
\end{aligned}
$$

The extraction of $\mathbf{r}_{1}^{i}$ et $\mathbf{r}_{2}^{i}$ can be realised as:

$$
\begin{aligned}
i & =\text { remainder }\left(t, n_{c}\right)+1 \\
\mathbf{r}_{1}^{i}(t) & =\mathbf{R}_{1}\left(1: n_{x}, i\right)(t) \\
\mathbf{r}_{2}^{i}(t) & =\mathbf{R}_{2}\left(n_{x}+1: n_{y} f, i\right)(t) .
\end{aligned}
$$

The computational complexity of this minimization technique is $\mathscr{O}\left(n_{\xi} n_{y} f\right)$ or $\mathscr{O}\left(n_{y}^{2} f^{2}\right)$ depending on the considered criterion (resp. $J_{I V}$ and $\breve{J}$ ). Other recursive subspace identification methods have similar complexities (Lovera et al., 2000).

Thus, a single minimization algorithm can be applied to two different cost functions in order to recursively estimate the propagator. Associated with the corresponding recursive MOESP update schemes, two new algorithms, named COPM and COIVPM, can then be proposed (cf. fig. 1).

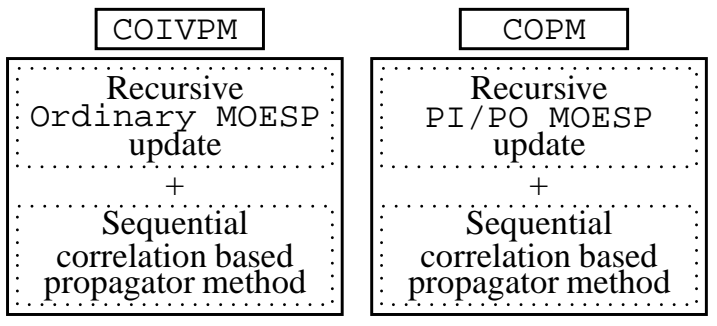

Fig. 1. COPM and COIVPM algorithms

\section{SIMULATION EXAMPLE}

In this section, a simulation example is proposed to show the performances of the developed algorithms. For that, consider the following $4^{\text {th }}$ order system:

$$
\begin{aligned}
\mathbf{x}(t+1) & =\left[\begin{array}{cccc}
0.85 & -0.4 & 0.52 & 0.3 \\
0 & -0.93 & -0.75 & -0.7 \\
0 & 0 & 0.95 & -0.4 \\
0 & 0 & 0 & 0.81
\end{array}\right] \mathbf{x}(t)+\left[\begin{array}{cc}
0 & 0 \\
0 & -0.86 \\
0.75 & 0 \\
0.6 & 0.3
\end{array}\right] \tilde{\mathbf{u}}(t) \\
\mathbf{y}(t) & =\left[\begin{array}{cccc}
0.7 & -0.8 & 0 & 0.6 \\
0 & 0 & 1.5 & -0.5
\end{array}\right] \mathbf{x}(t)+\left[\begin{array}{c}
0.3 \\
0.27
\end{array}\right] \mathbf{v}(t) \\
\mathbf{u}(t) & =\tilde{\mathbf{u}}(t)+\mathbf{f}(t)
\end{aligned}
$$

where $\tilde{\mathbf{u}}, \mathbf{v}$ and $\mathbf{f}$ are three independent white noises with respective variance $1,0.1$ and 0.01 . The construction parameters are chosen as:

$$
\begin{aligned}
f & =p=10 \\
\lambda(t) & =\lambda_{0} \lambda(t-1)+1-\lambda_{0}
\end{aligned}
$$

with $\lambda_{0}=\lambda_{0}=0.995$ and $\lambda_{\text {final }}=0.999$. The initial state space matrices are randomly generated.

In order to stress on the benefits of the COPM and COIVPM algorithms, it is interesting to compare them 


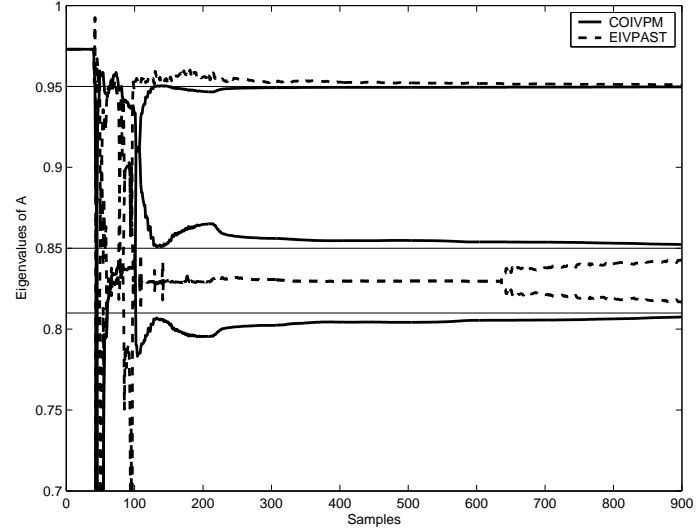

Fig. 2. Comparison of the estimated eigenvalues obtained with COIVPM and EIVPAST

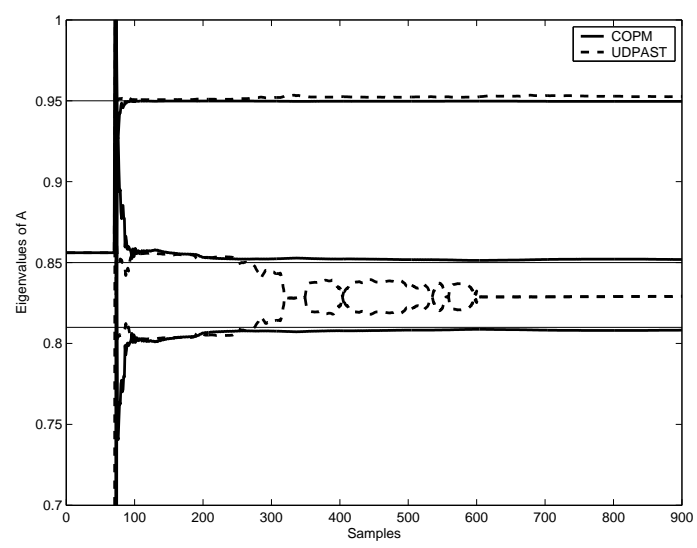

Fig. 3. Comparison of the estimated eigenvalues obtained with COPM and UDPAST

to other methods which have already shown their efficiency. The chosen techniques for this comparison are EIVPAST and UDPAST (Lovera et al., 2000). These one respectively use the Ordinary MOESP update and the PI/PO MOESP update schemes for the observation vector estimation step. An easy way to verify the accuracy of the estimated models consists in comparing the estimated poles obtained with the recursive identification algorithms with the theoretical one i.e. $0.95,0.85,0.81$ and -0.93 . This comparison is illustrated in figures 2 and 3. A zoom on the three largest poles is proposed. These curves clearly show that, contrary to EIVPAST and UDPAST, COPM and COIVPM are able to consistently estimate and distinguish close poles in a noisy environment.

\section{CONCLUSION}

Based on the Ordinary MOESP and on the PI / PO MOESP uptade schemes, two new recursive subspace identification methods respectively called COPM and COIVPM have been presented. These methods have in common to share a single algorithm to recursively estimate a basis of the observability subspace. The performances of these algorithms have been highlighted on a simulation example.

\section{REFERENCES}

Golub, G. H. and C. F. Van Loan (1996). Matrix computations. $3^{\text {rd }}$ ed.. John Hopkins University Press. Baltimore MD.

Gustafsson, T. (1998). Instrumental variable subspace tracking using projection approximation. IEEE Transactions on Signal Processing 46, 669-681.

Krim, H. and M. Viberg (1996). Two decades of array signal processing research: the parametric approach. IEEE Signal Processing Magazine 13, 67-94.

Ljung, L. (1999). System identification. Theory for the user. $2^{\text {nd }}$ ed.. PTR Prentice Hall Information and System Sciences Series. T. Kailath, Series Editor. Upper Saddle River.

Lovera, M. (2003). Recursive subspace identification based on projector tracking. In: The $13^{\text {th }}$ IFAC Symposium on System Identification. Rotterdam, The Netherlands.

Lovera, M., T. Gustafsson and M. Verhaegen (2000). Recursive subspace identification of linear and non linear Wiener state space models. Automatica 36, 1639-1650.

Mercère, G., S. Lecœuche and C. Vasseur (2003). A new recursive method for subspace identification of noisy systems: EIVPM. In: The $13^{\text {th }}$ IFAC Symposium on System Identification. Rotterdam, The Netherlands.

Mercère, G., S. Lecœuche and C. Vasseur (2004a). Robust adaptation of the propagator method to recursive subspace identification (in french). In: La Conférence International Francophone d'Automatique. Douz, Tunisia.

Mercère, G., S. Lecœuche and M. Lovera (2004b). Recursive subspace identification based on instrumental variable unconstrained quadratic optimization. International Journal of Adaptive Control and Signal Processing 18, 771-797.

Munier, J. and G. Y. Delisle (1991). Spatial analysis using new properties of the cross spectral matrix. IEEE Transactions on Signal Processing 39, 746-749.

Verhaegen, M. (1994). Identification of the deterministic part of MIMO state space models given in innovations form from input output data. Automatica 30, 61-74.

Verhaegen, M. and P. Dewilde (1992). Subspace model identification part 1: output error state space model identification class of algorithms. International Journal of Control 56, 1187-1210.

Viberg, M. (1995). Subspace based methods for the identification of linear time invariant systems. Automatica 31, 1835-1851.

Yang, B. (1995). Projection approximation subspace tracking. IEEE Transactions on Signal Processing 43, 95-107.

Yu, J. L. (2000). A novel subspace tracking using correlation based projection approximation. Signal Processing 80, 2517-2525. 